\title{
From Rouse dynamics to local relaxation: A neutron spin echo study on polyisobutylene melts
}

Cite as: J. Chem. Phys. 111, 6107 (1999); https://doi.org/10.1063/1.479907

Submitted: 15 February 1999 . Accepted: 06 July 1999 . Published Online: 24 September 1999

D. Richter, M. Monkenbusch, J. Allgeier, A. Arbe, J. Colmenero, B. Farago, Y. Cheol Bae, and R. Faust

\section{ARTICLES YOU MAY BE INTERESTED IN}

A Theory of the Linear Viscoelastic Properties of Dilute Solutions of Coiling Polymers

The Journal of Chemical Physics 21, 1272 (1953); https://doi.org/10.1063/1.1699180

Dynamics of entangled linear polymer melts: A molecular-dynamics simulation

The Journal of Chemical Physics 92, 5057 (1990); https://doi.org/10.1063/1.458541

Influence of stiffness on the dynamics of macromolecules in a melt

The Journal of Chemical Physics 106, 2469 (1997); https://doi.org/10.1063/1.473154

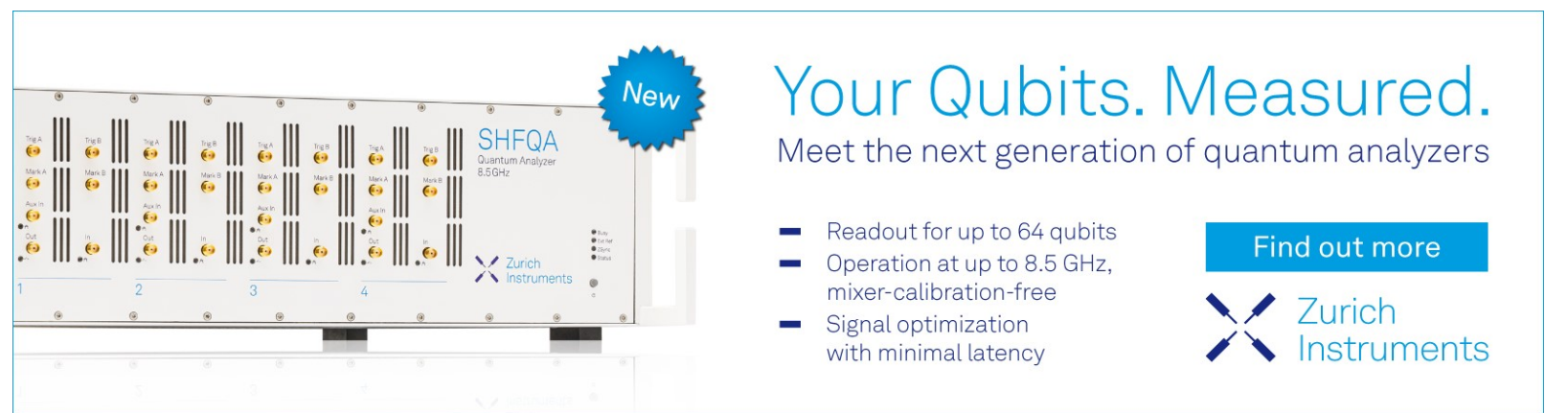

J. Chem. Phys. 111, 6107 (1999); https://doi.org/10.1063/1.479907

111,6107

(c) 1999 American Institute of Physics. 


\title{
From Rouse dynamics to local relaxation: A neutron spin echo study on polyisobutylene melts
}

\author{
D. Richter, M. Monkenbusch, and J. Allgeier \\ Institut für Festkörperforschung, Forschungszentrum Jülich, 52425 Jülich, Germany
}

\author{
A. Arbe and J. Colmenero \\ Departamento de Física de Materiales y Centro Mixto CSIC-UVP/EHU, Universidad del País Vasco, \\ Apartado 1072, 20080 San Sebastian, Spain \\ B. Farago \\ Institut Laue-Langevin, 156X, 38042 Grenoble Cedex, France
}

Y. Cheol Bae and R. Faust

Polymer Science Program, Department of Chemistry, University of Massachusetts-Lowell, One University Avenue, Lowell, Massachusetts 01854

(Received 15 February 1999; accepted 6 July 1999)

\begin{abstract}
We investigated the single chain motions of monodisperse polyisobutylene chains in the melt by neutron spin echo spectroscopy. Thereby a wide range in momentum space over a large dynamic range was covered. Motional processes from the center of mass diffusion, the Rouse dynamics to the more local relaxation processes which limit the validity of the standard Rouse model, were elucidated. The observed dynamic structure factors were analyzed in terms of relevant theoretical approaches addressing the limiting factors of the Rouse model. We found that other than claimed in the literature effects of local chain stiffness - they were treated in terms of the all rotational states model and a bending force model — cannot account for the experimental observations. It appears that additional damping effects related to an internal viscosity of the chain have to be involved, in order to explain the experimental results. (C) 1999 American Institute of Physics.
\end{abstract}

[S0021-9606(99)50537-0]

\section{INTRODUCTION}

The standard model for polymer chain dynamics in the melt considers the conformational entropy as the only source for restoring forces which stabilizes excursions from equilibrium. In its range of application this so-called Rouse model ${ }^{1}$ is very successful. Recently its limitations have been investigated in detail comparing computer simulations and neutron spin echo experiments on polyethylene chains. ${ }^{2}$ Only small deviations between the Rouse model on the one hand and the experimental dynamic structure factor and the computer simulations on the other hand were found.

Beyond that from a conceptual point of view the model has its limitations.

(1) At large distances, where long chain melt topological constraints cause entanglements leading to the reptation mechanism. This range is not of interest here.

(2) At shorter distances, where the simplifying assumptions of the Rouse model cease to be valid and the local chain structure comes into play. Locally the chain is stiff; this rigidity leads to deviations from the Rouse model. Furthermore, as a consequence of the rotational potentials, local relaxation mechanisms across the rotational barriers come into play leading to an internal viscosity. Even more locally, we approach the regime of the elemental relaxation processes, the $\alpha$ process and possibly higher order relaxations.

It is the aim of this paper to elucidate experimentally this crossover from the Rouse regime to the more local relaxation processes by neutron spin echo experiments.
The paper is structured as follows: Starting with the standard model for chain dynamics, the Rouse model, we present several theoretical approaches which deal with the influence of the local chain properties on the chain dynamics and derive the proper dynamic structure factors. We display the experimental procedures including a description of the polyisobutylene (PIB) sample preparation and characterization as well as the scattering experiments. Thereafter the results are presented and analyzed in terms of the different models outlined in Sec. II. Finally, we critically assess the outcome and relate to earlier work in the literature.

\section{THEORETICAL CONSIDERATIONS}

In recent years a number of different theoretical considerations were proposed in order to describe the dynamics of a polymer in a length scale regime, where the assumptions of the universal Rouse model cease to be valid. While in the Rouse regime the chain motion is governed by entropic forces at shorter scales the specific chain structure becomes important. Chain stiffness effects as well as intrachain friction processes become important. These influences were modeled by different authors and the corresponding papers are written in different terminologies. It is the aim of this section to present these approaches in a unified "language" and to make transparent the assumptions and physical consequences of these models. Neutron scattering allows one to investigate the length scale dependence of dynamic phenom- 
ena and is suited uniquely to study the above effects on their proper length scale. For each of the models, therefore, we will derive the corresponding dynamic structure factors.

In order to set a reference, we briefly introduce the main ingredients of the Rouse model. We refer to the conformational entropy, the resulting entropic forces, and the basic Langevin equation with its standard solution, including the segment-segment correlation functions which are the basis for the calculation of the dynamic structure factors. The different quantities will reappear in a later discussion of the various models and reference to the Rouse model will allow us to access the modifications due to those models.

Thereafter we begin with a presentation of two different models dealing with local chains stiffness. The first is the so-called all rotational states model (ARS model), ${ }^{3}$ which is equivalent to the better known rotational isomeric state model (RIS model) ${ }^{4}$ and which undertakes a mode description of the chain statistics. In the ARS model the characteristic ratio $C_{\infty}$ becomes mode dependent. Thereby the probability function for chain configurations and consequently the free energy is modified. We reformulate the Rouse equations in terms of this model thereby including stiffness effects. ${ }^{5,6}$ The second approach models the stiffness in terms of bending elasticity-fourth-order derivative with respect to the contour coordinate-in the Rouse equation. ${ }^{7}$ Again we give solutions for this modified Rouse equation and discuss the modifications of the dynamic structure factor.

In a third step we introduce the concept of internal viscosity, ${ }^{5,6}$ which deals with the additional dissipation of energy due to intrachain barriers. The internal viscosity force is derived from nonequilibrium thermodynamics considerations and leads to a memory term in the Rouse equation. The corresponding solutions consist of a two-mode relaxation process in terms of which the dynamic structure factor is formulated.

\section{A. The Rouse model as a reference}

The Rouse model may be considered as the standard model for polymer chain dynamics and is the starting point for most of the more frequently elaborated theories which refine this standard approach. In spite of its simplicity its general predictions have been verified to an astonishing detail on a molecular scale. ${ }^{8}$ In order to later dwell on the standard expressions of this model we briefly recall its physical basis and its solution.

The Rouse model treats the motion of a chain consisting of $N_{0}$ freely joint Gaussian segments in a heat bath. The conformation of such a Gaussian chain is described by a set of segment vectors along the chain $\{\mathbf{l}(n)\}$ with $\mathbf{I}(n)=\mathbf{R}(n)$ $-\mathbf{R}(n-1)$, where $\mathbf{R}(n)$ is the position vector of segment $n$. The conformational probability follows from the product of the $N_{0}$-independent Gaussian distribution factors for the single segments,

$$
W\{\mathbf{l}(n)\}=\prod_{n=1}^{N_{0}}\left(\frac{3}{2 \pi \mathbf{l}^{2}}\right)^{3 / 2} \exp \left[-3 \frac{\mathbf{l}^{2}(n)}{2 \mathbf{l}^{2}}\right],
$$

where $\mathbf{I}$ is the equilibrium segment length. The free energy $F$ of a Gaussian chain is entirely described by the conformational entropy $S=k_{B} \ln (W)$, where $k_{B}$ is the Boltzmann con- stant. This free energy $F$ exerts an entropic force on each segment which follows from the derivative of $F$ with respect to the positional coordinate. Due to inclusion of the entropic force the Langevin equation for segment motion assumes the form

$$
\zeta \frac{\partial x(n)}{\partial t}=\frac{3 k_{B} T}{\mathbf{l}^{2}}[x(n+1)-2 x(n)+x(n-1)]+f(n, t),
$$

where the first term on the right-hand side is the entropy force, and $f(n, t)$ is the stochastic force exerted by the heat bath. $\zeta$ is the segmental friction coefficient and the only parameter of the model. With the boundary conditions of force free ends this Rouse equation is readily solved by cosine Fourier transformation resulting in a spectrum of normal modes. The dispersion relation of the corresponding mode relaxation rates is obtained as

$$
\frac{1}{\tau_{p}}=2 W\left[1-\cos \left(\frac{p \pi}{N_{0}}\right)\right] \cong \frac{3 \pi^{2} k_{B} T}{\zeta \mathbf{l}^{2} N_{0}^{2}} p^{2}=\frac{p^{2}}{\tau_{R}}
$$

with the elementary Rouse relaxation rate $W=3 k_{B} T / \zeta 1^{2}$ and $\tau_{R}$ the so-called Rouse time, the longest relaxation time of the chain.

For the correlation functions of the Rouse modes one obtains $^{9}$

$\left\langle\widetilde{x}_{\alpha}(p, t) \widetilde{x}_{\beta}(q, 0)\right\rangle=\delta_{p q} \delta_{\alpha \beta} \frac{k_{B} T}{2 \zeta N_{0}} \tau_{p} \exp \left(-\frac{t}{\tau_{p}}\right) \quad(p, q>0)$.

Thereby $\widetilde{x}_{\alpha}(p, t)$ is the $\alpha$ component of the normal mode $p$; for $p=0$ the center of mass motion is obtained.

Scattering experiments measure the mean square segment correlation functions

$$
B(n, m, t)=3\left\langle[x(n, t)-x(m, 0)]^{2}\right\rangle .
$$

They are obtained by backtransformation of the normal coordinates into real space,

$$
\begin{aligned}
B(n, m, t)= & 6 D_{R} t+|m-n| \mathrm{I}^{2}+\frac{12 k_{B} T}{\zeta N_{0}} \sum_{p=1}^{N_{0}} \tau_{p} \cos \left(\frac{p \pi m}{N_{0}}\right) \\
& \times \cos \left(\frac{p \pi n}{N_{0}}\right)\left[1-\exp \left(-\frac{t}{\tau_{p}}\right)\right] .
\end{aligned}
$$

The factor $\tau_{p}$ in the sum results from the equipartition theorem associating each independent mode with the energy $3 k_{B} T / 2 ; D_{R}=k_{B} T / \zeta N_{0}=\left(1^{2} / 3 N_{0}\right) W$ is the Rouse diffusion coefficient.

By means of neutron scattering two different correlation functions may be accessed. In the case of coherent scattering all partial waves emanating from different scattering centers are capable of interference- the Fourier transform of the pair correlation function is measured. In contrast, in incoherent scattering the interferences from partial waves of different scatterers average to zero-the self-correlation function is obtained. Having calculated the mean square displacement functions the two dynamic structure factors in Gaussian approximation are readily formulated, 


$$
\begin{aligned}
& S_{\text {inc }}(Q, t)=\frac{1}{N_{0}} \sum_{n=1}^{N_{0}} \exp \left[-\frac{Q^{2}}{6} B(n, n, t)\right], \\
& S_{\text {coh }}(Q, t)=\frac{1}{N_{0}^{2}} \sum_{n, m=1}^{N_{0}} \exp \left[-\frac{Q^{2}}{6} B(n, m, t)\right] .
\end{aligned}
$$

\section{B. The influence of chain stiffness}

If we increase the scattering vector, $S(Q, t)$ becomes more sensitive to shorter distances along the chain. The assumption of Gaussian beads will cease to hold and we expect that the local stiffness of the chain will become significant. This can be dealt with in terms of a mode description of the chain statistics or by introducing explicitly a bending term into the Rouse equation.

\section{Mode description of the chain statistics}

In a real chain segment-segment correlations extend beyond nearest-neighbor distances. The standard model to treat the local statistics of a chain which includes the local stiffness would be the rotational isomeric state (RIS) ${ }^{4}$ formalism. For a mode description as is required for an evaluation of the chain motion it is more appropriate to consider the so-called all rotational state (ARS) model, ${ }^{3}$ which describes the chain statistics in terms of orthogonal Rouse modes. It can be shown that both approaches are formally equivalent and only differ in the choice of the orthonormal basis for the representation of statistical weights.

In the standard RIS model the end to end distance $R_{E}$ is connected with the characteristic ratio $C_{\infty}$ by $R_{E}^{2}=\mathrm{l}_{0}^{2} C_{\infty} N$, where $\mathbf{I}_{0}$ is the bond length and $N$ the number of bonds along the main chain. In the ARS approach the characteristic ratio becomes mode dependent. As Allegra et al. ${ }^{6}$ have shown, for polyolefines it can be well approximated by

$$
\begin{aligned}
C(q)= & \frac{2(1-c)^{1 / 2}\left(C_{\infty}-c\right)}{\left(C_{\infty}-c\right)^{2}+(1-c)^{1 / 2}-\left[\left(C_{\infty}-c\right)^{2}-(1-c)^{1 / 2}\right] \cos q} \\
& +c
\end{aligned}
$$

with $c \cong 0.4, C_{\infty}$ the characteristic ratio of the particular polyolefine, and $q=p \pi / N$. The mode-dependent characteristic ratios are bell-shaped curves peaked around $q=0$ with $C(0)=C_{\infty}$. If $\widetilde{\mathbf{I}}(q)$ is the Fourier transform of the segment vectors $\mathbf{l}(n)$, then $C(q)$ connects to the segment-segment correlation function by

$$
\frac{1}{N}\left\langle\widetilde{\mathbf{l}}(q) \widetilde{\mathbf{I}}^{*}(q)\right\rangle=C(q) \mathbf{l}_{0}^{2} .
$$

The higher the mode number, the smaller the square of the Fourier components is.

Using periodic boundary conditions and considering that a segment vector is given by the difference of adjacent position vectors $\mathbf{R}(n+1)-\mathbf{R}(n)$, the statistical average for $\left\langle\widetilde{\mathbf{R}}(q)^{2}\right\rangle$ in Fourier space becomes

$$
\left\langle\widetilde{\mathbf{R}}(q)^{2}\right\rangle=\frac{\left\langle\widetilde{\mathbf{I}}(q)^{2}\right\rangle}{4 \sin ^{2}\left(\frac{q}{2}\right)}=\frac{N \mathbf{l}_{0}^{2} C(q)}{4 \sin ^{2}\left(\frac{q}{2}\right)}\left\langle\widetilde{\mathbf{l}}(q)^{2}\right\rangle .
$$

With this equation in place we now may evaluate the statistical average for any distance $k=|h-j|$ along the chain, where $h$ and $j$ denote the position of chain segments. Replacing $\mathbf{I}^{2}$ by $\left\langle\widetilde{\mathbf{I}}(q)^{2}\right\rangle$ for such a chain the probability distribution for a chain conformation changes from Eq. (2.1) to

$$
\begin{aligned}
W(\{\widetilde{\mathbf{I}}(q)\})= & \prod_{\{q\}}\left(\frac{3}{2 \pi N C(q) \mathbf{l}_{0}^{2}}\right)^{3 / 2} \\
& \times \exp \left[-\sum_{\{q\}} 3 \frac{\mathbf{l}(q) \mathbf{I}^{*}(q)}{2 N C(q) \mathbf{l}_{0}^{2}}\right] .
\end{aligned}
$$

Like in the Rouse model from the probability distribution $W$ the free energy is obtained by taking the logarithm and finally the force exerted on a segment $h$ ( $x$ component) follows by taking the derivative of the free energy,

$$
f_{x}(h)=-\frac{\partial F}{\partial x(h)}=-\frac{3 k_{B} T}{N \mathbf{l}_{0}^{2}} \sum_{\{q\}} \frac{4 \sin ^{2}\left(\frac{q}{2}\right)}{C(q)} \tilde{x}(q) e^{-i q h} .
$$

The Fourier transformed position coordinate $\widetilde{x}(q)$ thereby is taken from Eq. (2.11). Having evaluated the force we now can reformulate the Rouse equation [Eq. (2.2)] introducing the new force term of Eq. (2.13). The Fourier transformed Rouse equation then reads

$$
\zeta \dot{\tilde{x}}(q)-\frac{3 k_{B} T}{\mathbf{l}_{0}^{2}} \frac{4 \sin ^{2}\left(\frac{q}{2}\right)}{C(q)} \widetilde{x}(q)=\widetilde{F}_{x}(q, t),
$$

where $\widetilde{F}_{x}$ denotes the $x$ component of the appropriate Fourier component of the random force. Comparing Eq. (2.14) with Eq. (2.2) we realize that the mode-dependent characteristic ratio $C(q)$ leads to a stiffening of the chain for higher $q$, where $C(q)$ drops [Eq. (2.9)] and consequently the spring constant increases. As a result the characteristic relaxation times are shortened compared to Eq. (2.3). We have

$$
\frac{1}{\tau_{q}}=\frac{12 k_{B} T \sin ^{2}\left(\frac{q}{2}\right)}{\zeta \mathbf{l}_{0}^{2} C(q)} \cong \frac{3 k_{B} T}{\zeta \mathbf{l}_{0}^{2} C(q)} q^{2} .
$$

The mean square displacements are calculated in the same spirit as for the simple Rouse model. If we consider the periodic chain transform we get

$$
\begin{aligned}
B(n, m, t) & =B(k, t) \\
& =\frac{6 k_{B} T}{N_{0} \zeta} \sum_{\{q\}} \tau_{q}\left[1-\cos (q k) \exp \left(-\frac{t}{\tau_{q}}\right)\right],
\end{aligned}
$$

where $k=|n-m|$. We note that because of the shorter relaxation times the weight at which the contribution of an eigenmode $q$ appears in the mean square displacement is reduced compared to the simple Rouse model. According to the equipartition theorem a stiffer spring leads to a smaller motional amplitude.

The dynamic structure factors now may be evaluated following Eq. (2.7) as above. 


\section{Chain dynamics with bending forces}

While in Sec. II A we started basically from a RIS description of the chain statistics, we now course grain and introduce the chain stiffness more generally in terms of a local rigidity which is exhibited by locally stiff chain molecules of Gaussian segments. Harnau et al. ${ }^{7}$ have shown that by a maximum entropy principle the partition function for such a chain can be formulated. Its evaluation leads to the correct average static properties of the Kratky-Porod wormlike chain. Here we consider such a chain and describe it by a continuous chain contour coordinate $-L / 2 \leqslant s \leqslant L / 2$, where $L$ is the overall contour length and $s$ the contour coordinate. $L$ relates to the number of chain bonds $N$ and the bond length $\mathbf{l}_{0}$ by $L=N \mathbf{l}_{0} \sin (\theta / 2)$, where $\theta$ is the bond angle. The stiffness is introduced into the continuous form of the Rouse equation (2.2) (the second-order difference term becomes second derivative) by a fourth-order derivative with respect to the contour coordinate

$\gamma \frac{\partial}{\partial t} \mathbf{R}(s, t)+\epsilon \frac{\partial^{4}}{\partial s^{4}} \mathbf{R}(s, t)-2 \nu \frac{\partial^{2}}{\partial s^{2}} \mathbf{R}(s, t)=\mathbf{f}(s, t)$,

with $\gamma=\zeta / l_{0} \sin \theta / 2$ the friction per unit length, $\epsilon=3 k_{B} T / 4 \tilde{p}$, and $\nu=3 \tilde{p} k_{B} T / 2 ; \tilde{p}$ relates to the persistence length of the chain and may be connected to $C_{\infty}$, for example, by the expression for the end to end distance of a wormlike chain $\left\langle R_{E}^{2}\right\rangle=L / \widetilde{p}-\left(1-e^{-2 \tilde{p} L}\right) / 2 \widetilde{p}^{2}$ and the corresponding expression for a RIS chain. For large $N, \tilde{p}$ $\cong \sin (\theta / 2) / C_{\infty} \mathbf{l}_{0}$ holds. With the appropriate boundary conditions of force free ends a normal mode analysis may be performed. ${ }^{7}$ From that analysis characteristic relaxation times are obtained as

$$
\frac{1}{\tau_{p}^{b}}=\frac{3 k_{B} T}{\gamma}\left(\tilde{p} \alpha_{p}^{2}+\frac{\alpha_{p}^{4}}{4 \tilde{p}}\right) .
$$

The coefficients $\alpha_{p}$ are obtained from transcendent equations [Eq. (2.25) in Ref. 7]. For $\epsilon=0$, Eq. (2.17) coincides with the Rouse equation [Eq. (2.2)]. For large $L \tilde{p}$ the coefficients $\alpha_{p}$ are very close to $\alpha_{p} \cong p \pi / L$. With this approximation Eq. (2.18) becomes

$\frac{1}{\tau_{p}^{b}}=\frac{W \pi^{2}}{N^{2}}\left[p^{2}+\frac{\pi^{2} p^{4} C_{\infty}^{2}}{4 N^{2} \sin (\theta / 2)}\right]=\frac{W \pi^{2}}{N^{2}}\left(p^{2}+\alpha_{B} p^{4}\right)$.

Equation (2.19), in its first part, agrees with the Rouse result of Eq. (2.3). The term proportional to $p^{4}$ is the correction due to rigidity effects. For higher mode numbers $p$ the relaxation rates $\left(\tau_{p}^{b}\right)^{-1}$ are increasingly accelerated. Furthermore, because of the equipartition theorem [see, e.g., Eq. (2.4)] the amplitude of the corresponding correlator will diminish. We notice that under these conditions also the eigenfunctions of Eq. (2.17) become very similar to those of the Rouse problem. In a first-order approximation, therefore, the mean square displacements [Eq. (2.6)] may be calculated by replacing the characteristic relaxation times $\tau_{p}=\tau_{R} / p^{2}$ by $\tau_{p}^{b}$. With these new expressions for the mean square displacements we then may evaluate the dynamic structure factor following Eq. (2.7). Using the same approach as for the Rouse model [Eq. (2.6)], we also may calculate the full solutions.

\section{Internal viscosity}

The description of the chain dynamics in terms of the Rouse model is not only limited by the local stiffness effects but also by local dissipative relaxation processes like jumps over the barrier in the rotational potential. Thus, in order to extend the range of description, a combination of the modified Rouse model with a simple description of the rotational jump processes is sought. Allegra et al. ${ }^{5,6}$ introduced an internal viscosity as a force which arises due to a departure from configurational equilibrium. Thereby, the rotational relaxation processes are described by one single relaxation rate $\tau_{0}$. From an expression for the difference in free energy due to small excursions from equilibrium an explicit expression for the internal viscosity force in terms of a memory function is derived. The internal viscosity force $\phi_{k}$ acting on the $k$ th backbone atom becomes

$$
\begin{aligned}
\phi_{k}(t)= & \mp \frac{i V}{N} \int_{-\infty}^{t} \exp \left[-\frac{\left(t-t^{\prime}\right)}{\tau_{0}}\right] \\
& \times\left[\sum_{\{q\}} \frac{3 k_{B} T}{C(q) l_{0}^{2}} \widetilde{x}(q, t) \sin (q) e^{-i q k}\right] d t^{\prime} .
\end{aligned}
$$

Thereby, $V$ is a numerical constant on the order of 1 . The sign describes the direction of the net force which is dictated by the direction of strain propagation along the chain. After Fourier transformation Eq. (2.20) may be introduced into the modified Rouse equation [Eq. (2.14)] yielding

$$
\begin{aligned}
& \frac{12 k_{B} T}{C(q) \mathbf{l}_{0}^{2}} \sin ^{2}\left(\frac{q}{2}\right) \tilde{x}(q, t) \pm i V \frac{3 k_{B} T}{C(q) \mathbf{l}_{0}^{2}} \sin (q) \int_{-\infty}^{t} d t^{\prime} \\
& \quad \times \exp \left[-\frac{\left(t-t^{\prime}\right)}{\tau_{0}}\right] \dot{x}\left(q, t^{\prime}\right)+\zeta \dot{\tilde{x}}(q, t)=\widetilde{F}_{x}(q, t) .
\end{aligned}
$$

The homogeneous Eq. (2.21) is solved by a decaying traveling wave

$$
\tilde{x}(q, t)=\tilde{x}_{0}(q) \exp \left\{\left[i \omega(q)-\frac{1}{\tau(q)}\right] t\right\},
$$

with

$$
\omega(q)+\frac{i}{\tau(q)}=\frac{A(q) \mp \sqrt{A^{2}(q)+B(\omega)}}{2 \zeta \tau_{0}},
$$

where

$$
\begin{aligned}
& A(q)=V \frac{3 k_{B} T}{\mathbf{l}_{0}^{2}} \tau_{0} \sin (q)-i\left[\zeta+2 \frac{12 k_{B} T}{\mathbf{l}_{0}^{2} C(q)} \tau_{0} \sin ^{2}\left(\frac{q}{2}\right)\right], \\
& B(q)=8 \frac{12 k_{B} T}{\mathbf{l}_{0}^{2} C(q)} \zeta \tau_{0} \sin ^{2}\left(\frac{q}{2}\right) .
\end{aligned}
$$

The eigenvalue Eq. (2.23a) leads to two branches of the relaxation time $\tau$ and the propagation frequency $\omega$. For experimental parameters taken from the later data evaluation Fig. 1 displays the dependence of the relaxation times on the mode number $p=q N / \pi$. For comparison also the solution $\tau_{q}$ of the ARS-Rouse equation-Eq. (2.15)—is displayed. While at low $p$ the solution for the longer relaxation time $\tau_{1}$ coincides with that of the modified Rouse equation at higher $p$, 


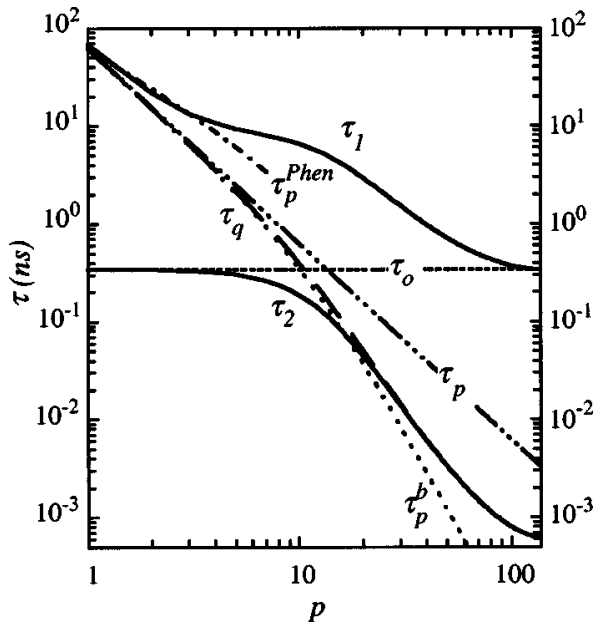

FIG. 1. Mode-number dependence of the relaxation times $\tau_{1}$ and $\tau_{2}$ (solid lines). The dashed-dotted line shows the relaxation time $\tau_{p}$ in the Rouse model [Eq. (2.3)]. The horizontal dashed line displays the value of $\tau_{0}$. The dashed and the dotted lines represent the relaxation time when the influence of the chain stiffness is considered: mode description of the chain statistics $\tau_{q}$ [dashed, Eq. (2.15)] and bending force model $\tau_{p}^{b}$ [dotted, Eq. (2.19)]. The behavior of the relaxation time $\tau_{p}^{\text {phen }}$ used in the phenomenological description is also shown for the lowest modes (see the text).

such a coincidence is observed for the shorter relaxation time $\tau_{2}$. In between both modes repel each other and for small and large $p$ reach the value of $\tau_{0}$. The multidot-dashed line represents the Rouse model. We realize that for small $p$ the Rouse relaxation times agree well with $\tau_{q}$, while for the higher mode number due to the stiffness the relaxation times are significantly decreased. Finally we also include the relaxation times of the bending model $\tau_{p}^{b}$ (the dotted line). These times decrease even more strongly than those of the ARS model.

The mean square segment displacements which are the key ingredient for a calculation of the dynamic structure factor are obtained from a calculation of the eigenfunctions of the differential equation (2.21). After retransformation from Fourier space to real space $B(k, t)$ is given by Eq. (41) of Ref. 5. For short chains the integral over the mode variable $q$ has to be replaced by the appropriate sum. Finally, for observation times $t \gg \tau_{0}$ the mean square displacements can be expressed in terms of eigenvalue $\tau_{1}$ only. Thereby a significant simplification of the expression for the mean square displacements is achieved (see Ref. 6).

\section{EXPERIMENT}

\section{A. Samples}

\section{Sample preparation}

The deuterated isobutylene (IB) monomer (CDN Isotopes, 99.6 at. \% deuterium) was purified by passing the gaseous monomer through a column packed with $\mathrm{CaH}_{2}$ and condensing the material at $-80^{\circ} \mathrm{C}$. It was further dried by distillation from $\mathrm{CaH}_{2}$. All other chemicals and solvents were purified as described previously. ${ }^{10}$ The living cationic polymerizations of the hydrogenous and the deuterated IB monomers were carried out under a dry nitrogen atmosphere $\left(\left[\mathrm{H}_{2} \mathrm{O}\right]<1.0 \mathrm{ppm}\right)$ in a MBraun $150-\mathrm{M}$ glovebox using the 2-chloro-2,4,4-trimethylpentane (TMPCl)/TiCl4/hexane: methyl chloride $(60: 40, \mathrm{v}: \mathrm{v})$ system in the presence of the proton trap 2,6-di-tert-butylpyridine (DTBP). Hexane, the initiator TMPCl, and DTBP were charged to the reactor at room temperature. The temperature was lowered to $-80^{\circ} \mathrm{C}$ and methyl chloride as well as one-third of the monomer was added. The polymerization was started by the addition of the coinitiator $\mathrm{TiCl}_{4}$. In $15 \mathrm{~min}$ intervals the rest of the monomer was added in two increments. The following concentrations were used: $[\mathrm{TMPCl}]=0.003 \mathrm{M},\left[\mathrm{TiCl}_{4}\right]=0.027 \mathrm{M}$ (for the hydrogenous monomer), and $\left[\mathrm{TiCl}_{4}\right]=0.033 \mathrm{M}$ (for the deuterated monomer), $[\mathrm{DTBP}]=0.003 \mathrm{M}$, and $[\mathrm{IB}]=0.21 \mathrm{M}$. A higher concentration of $\mathrm{TiCl}_{4}$ was used in case of deuterated IB due to a higher level of protic impurities in the monomer. In order to achieve a low molecular weight distribution $M_{w} / M_{n}$ the incremental monomer addition technique was employed. The polymerization was continued for $1.5 \mathrm{~h}$ after the last monomer addition and quenched with chilled methanol. The terminal chloro-group in the polyisobutylene was removed by dehydrochlorination according to the procedure reported by Kennedy et al. ${ }^{11}$

\section{Characterization}

The degrees of polymerization (DP) and $M_{w} / M_{n}$ of the polyisobutylenes were measured using a Waters highperformance liquid chromatography (HPLC) system equipped with a model 510 HPLC pump, model 250 dual detector (refractometer/viscometer, Viscotek), model 486 tunable UV/VIS detector, and model 712 sample processor. Five Ultrastyragel gel permeation chromatography columns were used, the eluant was THF, and the flow rate $1.0 \mathrm{ml} / \mathrm{min}$.

The DP and $M_{w} / M_{n}$ values found for the polymers were in case of the hydrogenous polyisobutylene $\mathrm{DP}=69$ and $M_{w} / M_{n}=1.06$ and in case of the deuterated version DP $=70$ and $M_{w} / M_{n}=1.05$.

\section{B. Dynamic structure factor measurements}

\section{The neutron spin echo technique}

Neutron spin echo (NSE) is the most suitable technique for exploring dynamical processes in a wide momentum transfer $(Q)$ range with very high energy resolution. ${ }^{11}$ This is achieved by coding the energy transfer in the scattering process for each neutron individually into its spin rotation. The Larmor precession of the neutron spin in an external magnetic field uses the neutron spin as an internal clock. In this way, the application of precession magnetic fields before and after the scattering event results in a polarization of the neutron that depends only on the velocity difference of each neutron individually, irrespective of its initial velocity. Energy resolution and monochromatization of the incident beam are thus decoupled, and resolutions in energy of the order of $10^{-5}$ can be achieved with an incident neutron spectrum of $20 \%$ bandwidth.

The NSE experiment consists in the study of the final polarization of the neutron beam, which is directly related to the normalized intermediate scattering function $S(Q, t)$ / $S(Q, 0) .{ }^{12}$ The time $t$ depends on the wavelength $\lambda$ of the neutron, the precession field $B$, and the length of the preces- 
sion field $L, t \propto B L \lambda^{3}$. Thus the time evolution of the structure factor can be followed varying $B$. For a certain range of applicable fields, the accessible time range of the instrument can be modified by choosing a different wavelength $\lambda$, and therefore for a given $Q$ value it is possible to explore different time windows by simultaneously varying the scattering angle $2 \theta$ and the selected wavelength. This allows the observation of a given process through a large $Q$ range in the best possible dynamic interval for each $Q$ value, which is essential for the purpose of this study.

On the other hand, the spin echo signal can be diminished or even reversed by neutrons which are spin-flipped by the sample, if some or all the scattering is spin incoherent. The measured "spin up," $u$, and "spin down," $d$, are then related to the coherent and incoherent scattering intensities $I_{\text {coh }}$ and $I_{\text {inc }}$ through

$$
\begin{aligned}
& u=I_{\mathrm{coh}}+\frac{1}{3} I_{\mathrm{inc}}+\frac{1}{f}\left(\frac{2}{3} I_{\mathrm{inc}}\right), \\
& d=\frac{2}{3} I_{\mathrm{inc}}+\frac{1}{f}\left(I_{\mathrm{coh}}+\frac{1}{3} I_{\mathrm{inc}}\right),
\end{aligned}
$$

where $f$ is the flipping ratio of the flippers $(f=25)$. The signal, given by the difference $u-d$, therefore has contributions from both the coherent and the incoherent scattering,

$$
u-d=\left(I_{\mathrm{coh}}-\frac{1}{3} I_{\mathrm{inc}}\right)\left(1-\frac{1}{f}\right)
$$

and the result of the neutron spin echo measurement becomes

$$
\frac{S(Q, t)}{S(Q, 0)}=\frac{I_{\mathrm{coh}} \widetilde{S}_{\mathrm{coh}}(Q, t)-\frac{1}{3} I_{\mathrm{inc}} \widetilde{S}_{\mathrm{inc}}(Q, t)}{I_{\mathrm{coh}}-\frac{1}{3} I_{\mathrm{inc}}},
$$

thus $\widetilde{S}_{\text {coh }}(Q, t)$ and $\widetilde{S}_{\text {inc }}(Q, t)$ are the normalized coherent and incoherent scattering functions, respectively.

The last feature of NSE we would like to highlight is that, since NSE directly measures in the time domain, resolution corrections consist of simple division of the spectra by the resolution function.

In this work we used the spectrometer IN11 at the Institute Laue-Langevin (ILL) in Grenoble, France. IN11 is well suited for extending the study to short times, since $\lambda$ may be varied between 12 and $4 \AA$.

\section{Single chain dynamic structure factor}

If some protonated chains are dissolved in a deuterated matrix, the scattering intensity results from the interfering partial waves originating from the different monomers of the same chain. ${ }^{8}$ Thus NSE measures the pair-correlation function for the dynamics of a single chain. Our sample $(2 \mathrm{~mm}$ thickness in a tight niobium sample holder) contained $10 \%$ protonated chains of PIB. In order to extend our study to the maximum $Q$-range possible, we chose a temperature high enough $(470 \mathrm{~K})$ where we could expect to observe some decay of the structure factor in the accessible time window even for the lowest accessible $Q$ values. We also performed

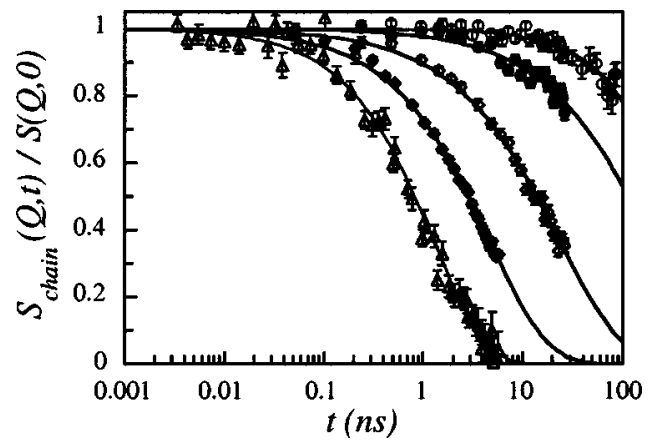

FIG. 2. Intermediate dynamic structure factor of the single chain dynamics measured for PIB on IN11 at $470 \mathrm{~K}$ and different $Q$ values: $0.04 \AA^{-1}(\bigcirc)$, $0.08 \AA^{-1}(\bullet), 0.15 \AA^{-1}(\diamond), 0.25 \AA^{-1}(\diamond)$, and $0.40 \AA^{-1}(\triangle)$. The solid lines display fits with stretched exponential functions.

measurements at $390 \mathrm{~K}$, the highest temperature at which the collective dynamics on PIB has been previously investigated. ${ }^{13}$ Moreover, we used three different wavelengths depending on the $Q$ range investigated for selecting the most suitable observation time range. These were $\lambda$ $=11.86,8$, and $4.8 \AA$, which yielded time windows of

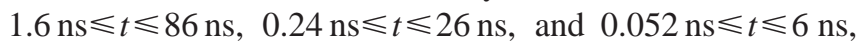
respectively. In addition, at the highest $Q$ values investigated we also performed measurements with a short spin echo setup in order to extend the study to even shorter times, covering $0.003 \mathrm{~ns} \leqslant t \leqslant 5.1 \mathrm{~ns}$ with $\lambda=4.8 \AA$. The $Q$ values studied were [in brackets, the $\lambda(\AA)$ used]: $0.03 \AA^{-1}(11.86)$, $0.04 \AA^{-1}$ (11.86 and 8), $0.06 \AA^{-1}$ (8), $0.08 \AA^{-1}(8), 0.10 \AA^{-1}$ (8), $0.15 \AA^{-1}$ (8), $0.20 \AA^{-1}$ (8 and 4.8), $0.25 \AA^{-1}$ (4.8), 0.30 $\AA^{-1}$ (4.8), $0.35 \AA^{-1}$ (4.8), $0.40 \AA^{-1}$ (4.8 short spin echo), and $0.50 \AA^{-1}$ (4.8, short spin echo). Typical measuring times were $8 \mathrm{~h}$ for $\lambda=11.86 \AA$, between 1 and $2 \mathrm{~h}$ for 8 and $4.8 \AA$, and $4 \mathrm{~h}$ for the two highest $Q$ values. The background from the $\mathrm{Nb}$ container and the scattering from the deuterated matrix were measured at each wavelength. At each $Q$ value and temperature the matrix data were taken with similar statistics as those from the sample. Careful measurements of the values of $u$ and $d$ and the transmissions of sample and matrix allowed us to calculate the proper factors for the subtraction of the matrix and container scattering. These corrections became more and more important with increasing $Q$, because the scattering from the sample was falling below the scattering from the matrix for $Q \geqslant 0.4 \AA^{-1}$. Resolution corrections were performed by using Graphite for $Q<0.4 \AA^{-1}$ and TiZi for $Q \geqslant 0.4 \AA^{-1}$.

\section{RESULTS}

Some of the measured spectra at $470 \mathrm{~K}$ are shown in Fig. 2. The data are of excellent quality and it is worthwhile to point out the huge time interval explored by IN11 (almost five decades). This allows an observation of the chain dynamics in an experimental window $0.03 \AA^{-1} \leqslant Q$ $\leqslant 0.5 \AA^{-1}$.

A simple inspection of the spectra reveals nonexponential behavior for most of them. As a first phenomenological description we may use Kohlrausch-Williams-Watts 

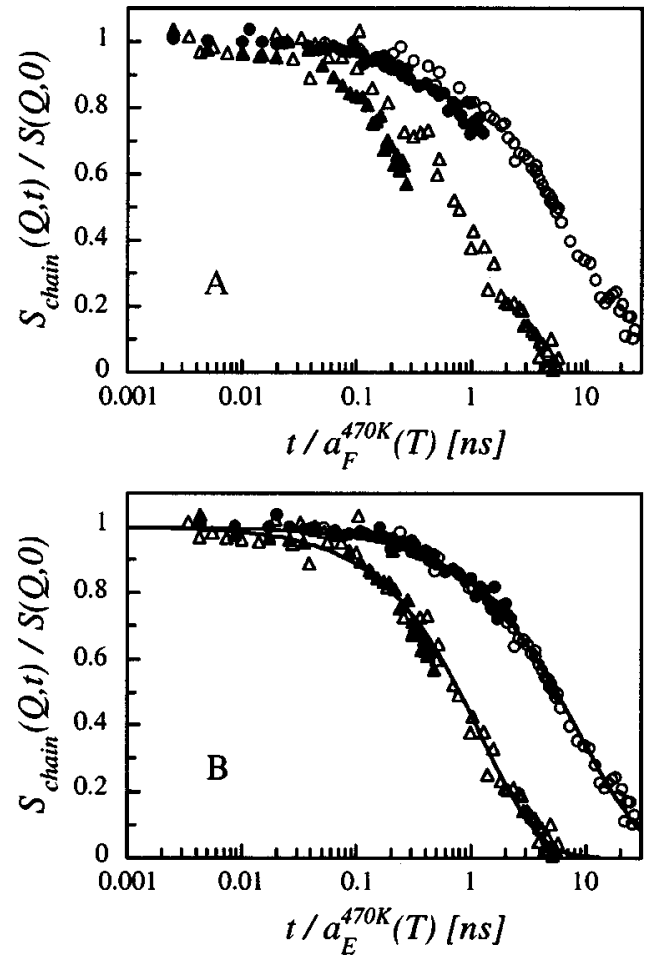

FIG. 3. Scaling representation of the NSE data corresponding to the single chain dynamics at $470 \mathrm{~K}$ (open symbols) and $390 \mathrm{~K}$ (closed symbols) for two $Q$ values: $0.2 \AA^{-1}$ (circles) and $0.4 \AA^{-1}$ (triangles). The rheological shift factor of Ferry [Eq. (4.2)] has been used in (a), whereas an experimentally determined shift factor brings the data together in (b). Solid lines show the fit with stretched exponential functions to the resulting master curves.

(KWW) or stretched exponential functions to parametrize the data,

$$
S(Q, t)=\exp \left[-\left(\frac{t}{\tau_{\mathrm{KWW}}(Q)}\right)^{\beta(Q)}\right],
$$

where $\beta(Q)$ is the stretching exponent or shape parameter characterizing the deviations from the single exponential decay and $\tau_{\mathrm{KWW}}(Q)$ is the KWW relaxation time. As can be seen in Fig. 2, this functional form very accurately describes all the experimental spectra.

Recently, investigating the collective dynamics at the maximum of the static structure factor $S(Q)$ it was found that the temperature dependence of the NSE data followed very well the rheological shift factors $a_{F}(T)$ determined by Ferry et al. ${ }^{14}$

$$
\log _{10}\left[a_{F}(T)\right]=-\frac{9.08(T-298.2 k)}{209 k+T-298.2 k},
$$

which was determined within an overlapping temperature window. In order to check whether this law also gives account for the temperature dependence of the measured chain dynamic structure factor, we shifted the time scale of the 390 $\mathrm{K}$ spectra according to Ferry's law taking as reference the temperature of $470 \mathrm{~K}$. Equation (4.2) yields a ratio of 21 between the scales at 390 and $470 \mathrm{~K}$. The result of such a scaling is shown in Fig. 3(a) for two $Q$ values. Obviously, as can be seen in Fig. 3(a), the extrapolation of the rheological results to the high temperature range does not account for the

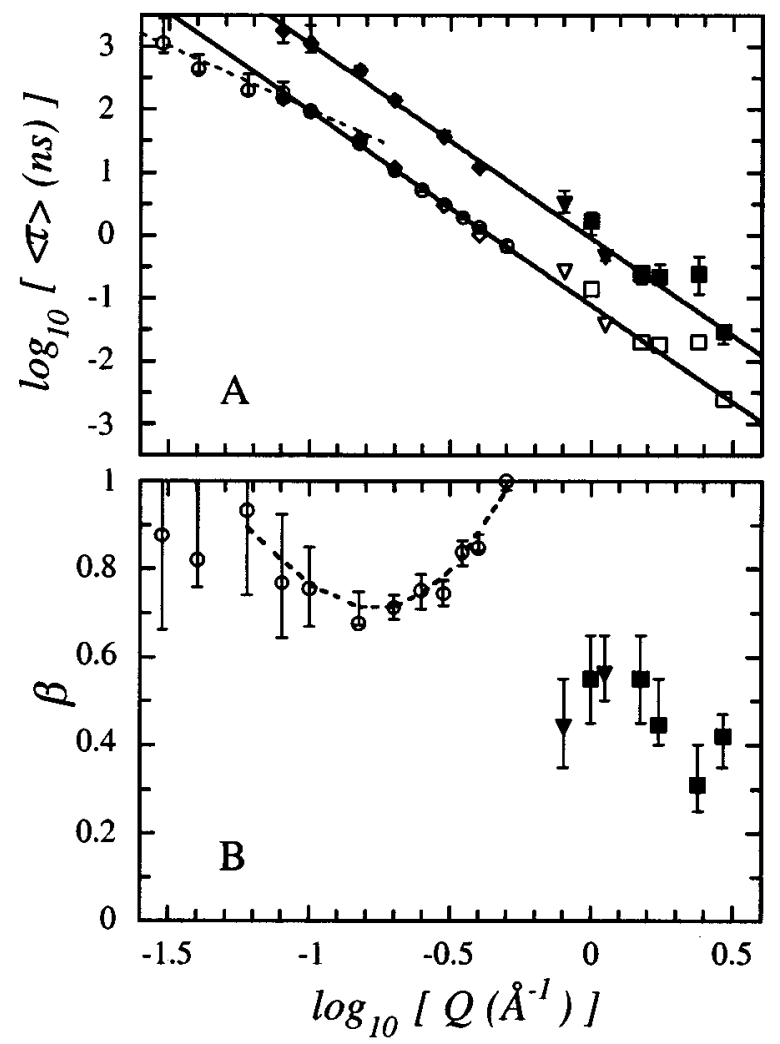

FIG. 4. Momentum transfer dependence of the average relaxation time $\langle\tau\rangle$ (a) and shape parameter $\beta$ (b), obtained from the description of the NSE data by means of a Kohlrausch-Williams-Watts function: spectra corresponding to the single chain dynamics at $390 \mathrm{~K}(\checkmark)$ and $470 \mathrm{~K}(\bigcirc)$, and to

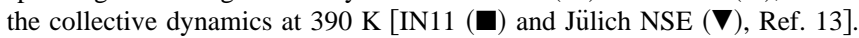
The results of applying the empirical shift factor found to the data at $390 \mathrm{~K}$ in (a) are shown by the corresponding open symbols. Solid lines are the fit to a power law of the characteristic times above $0.1 \AA^{-1}$. The dotted line in (a) represents a $Q^{-2}$ law. The dotted line in (b) is a smoothing curve characterizing the behavior of the chain data.

observed temperature dependence. Instead of the factor 21 deduced from Ferry's law, a shift factor of approximately 12 joins the spectra originating from these two temperatures [Fig. 3(b)]. Within the experimental uncertainty a similar temperature dependence is found over the full $Q$ range investigated. Since the superposition of the spectra is fairly good, we conclude that the shape of the dynamic structure factor does not significantly depend on temperature.

In order to compare characteristic times corresponding to relaxation functions with different shapes, we may use the average characteristic time $\langle\tau\rangle$ of the relaxation, defined as

$$
\langle\tau\rangle=\int_{0}^{\infty} S(Q, t) d t
$$

which in terms of a relaxation time distribution would correspond to the first moment of this distribution. In the case of a KWW function $\langle\tau\rangle$ relates to $\tau_{\mathrm{KWW}}$ through

$$
\langle\tau\rangle=\frac{\Gamma\left(\frac{1}{\beta}\right)}{\beta} \tau_{\mathrm{KWW}} .
$$

Figure 4(a) displays the dependence of $\langle\tau\rangle$ on momentum transfer $Q$, and Fig. 4(b) presents the corresponding re- 
sults for the shape parameter $\beta$. Figures 4(a) and 4(b) also include recently published results from the collective chain dynamics in a PIB melt. Here the data displayed for $470 \mathrm{~K}$ are a result of applying the experimental shift factor to the $390 \mathrm{~K}$ results. Two remarkable features stand out: (i) Surprisingly, the average relaxation times appear to follow a single power law starting from nearly the lowest $Q$ values up to $Q \approx 3.0 \AA^{-1}$. Thereby, the change over from the single chain dynamics to the collective response does not affect the observed $Q$ dependence. (ii) While the relaxation of the single chain tends to a Debye function for higher $Q$ values, the pair correlation function shows a markedly nonexponential form, which evidently stretches the more the farther the considered $Q$ deviates from the maximum of the main peak of $S(Q)\left[Q_{\max } \approx 1.0 \AA^{-1}\right]{ }^{13}$

The result pointed out in (i) is by no means trivial. At $390 \mathrm{~K}$ experimental data corresponding to both the chain dynamics and the collective dynamics are available. The error bars have been carefully estimated in both cases considering the changes in the deduced time scales when different shapes (KWW functions with $\beta$ values encompassing the experimental data with their error bars) are considered. The absolute values of the average time at this temperature seem to be very similar for both dynamics in the experimental crossover region, and they follow a continuous $Q$ dependence.

In order to study in detail the $Q$ dependence of $\langle\tau\rangle$, we have focused our attention on the $470 \mathrm{~K}$ data. At this temperature more accurate information can be extracted, because the decay is faster and better centered in the dynamical NSE window. A crossover to the center of mass diffusion appears to be observed for $Q$ values below $0.08 \AA^{-1}$ (note the bending of $\langle\tau\rangle$ toward a $Q^{-2}$ dependence). Above this $Q$ value, the average time can be perfectly described by the power law

$$
\langle\tau\rangle_{\text {chain }}^{470 \mathrm{~K}}(\mathrm{~ns})=0.077 Q\left(\AA^{-1}\right)^{-3.1} .
$$

The same $Q$ exponent also describes the $390 \mathrm{~K}$ data, resulting in the expression

$$
\langle\tau\rangle_{\text {chain }}^{390 \mathrm{~K}}(\mathrm{~ns})=0.90 Q\left(\AA^{-1}\right)^{-3.1} .
$$

The related shift factor amounts to 11.7 and is very close to the empirical value found from Fig. 3(b). The goodness of the description of $\langle\tau\rangle$ by Eqs. (4.5) and (4.6) can be appreciated in Fig. 4(a). The astonishing finding is that the extrapolations of these power laws are close to the average times obtained for the collective dynamics in the higher $Q$ range.

Concerning the collective dynamics, we would like to note that the observance of a power law even across the peak region at the static structure factor $\left(Q \approx 1.0 \AA^{-1}\right)$ contradicts the expectation of a deGennes narrowing ${ }^{15}$ in this range. New experiments with higher $Q$ resolution rectify this already previously noted deficiency in showing a definite modulation of $\langle\tau(Q)\rangle$ around $S\left(Q_{\max }\right) .{ }^{16}$ We also point out an apparent modulation of the shape parameter with $S(Q)$ which indicates itself in Fig. 4(b). In the $Q$ range close to the intrachain peak of $S(Q)\left(Q \approx 3.0 \AA^{-1}\right)$, this modulation could be due to the contribution of the Johari-Goldstein $\beta$ relaxation. ${ }^{13}$

\section{ANALYSIS OF THE DATA}

Beyond the phenomenological description of the spectra presented in Sec. IV, the analysis of the data in terms of the different approaches explained in Sec. II was performed. We tried to get the most detailed information about the mechanism driving the dynamics of the single chain. A careful comparison of the different model predictions with the experimental data was performed, taking into account the coherent and incoherent contributions to the spectra. Though small, the amount of incoherent scattering $I_{\text {inc }}$, together with the coherent intensity $I_{\text {coh }}$, can be determined from the "spin up" and "spin down" measurements at each $Q$ value [see Eq. (3.1)]. Since both the pair and the self-correlation functions can be calculated in terms of Eq. (2.7) for all the models we deal with, the NSE signal of the single chain dynamics could be fitted taking into account the correction given by Eq. (3.3).

We begin with the chain parameters necessary for an analysis of the data in terms of the different models presented in Sec. II. From the degree of polymerization (DP $=69$ ) we obtain for the number of main chain bonds $N$ $=138$. The calculation of $\mathbf{I}$ needs knowledge of the chain dimensions from small angle neutron scattering (SANS) measurements. In Ref. 17 the relation between the unperturbed end-to-end distance $R_{E}$ and the molecular weight $M_{n}$ is given by $R_{E}^{2}=0.570 M_{n}$ for PIB. Taking into account that $M_{n}=3870$, it follows $C_{\infty}=6.73$ and with $\mathbf{l}_{0}=1.54 \AA$, I $=4.0 \AA$ is found.

In the following the analysis of the data is presented.

\section{A. The Rouse model}

For $Q R_{E} \ll 1$, only the overall diffusion of the chain molecules is observed - the internal modes of the chain relaxation do not influence the dynamic structure factor. In the case of short length chains like the ones here studied, this $Q$ regime is relatively easily accessed with NSE. In fact, the results shown in Sec. IV [Figs. 4(a) and 4(b)] suggest the observation of such translational diffusion below $Q$ $=0.08 \AA^{-1}$. In this $Q$ regime an approximate Debye-like behavior was found with a $Q$ dispersion of the average time close to a $Q^{-2}$ law. In order to study in more detail the shape of the measured dynamic structure factor, in Fig. 5(a) we display the time dependence in a semilogarithmic representation. The solid line corresponds to a single exponential decay. This perfectly describes the decay of the data, though it is shown that, e.g., a KWW with $\beta=0.83^{2}$ would also account for the experimental behavior. Unfortunately, the statistics of the data does not allow a more accurate determination of the functional shape. Assuming a single exponential form as expected for translational diffusion, the fit to the $470 \mathrm{~K}$ spectra at 0.03 and $0.04 \AA^{-1}$ results in a value of $1.54 \pm 0.16 \AA^{2} \mathrm{~ns}^{-1}$ for the diffusion coefficient $D_{R}$. In Fig. 5 (b) we display the mean square center of mass displacement

$$
\left\langle\left[x_{0}(t)-x_{0}(0)\right]^{2}\right\rangle=-\frac{6 \ln \left[S_{\text {chain }}(Q, t)\right]}{Q^{2}}
$$

[see Eqs. (2.5)-(2.7)] combining data from different momentum transfers. At higher $Q$ values contributions of the inter- 


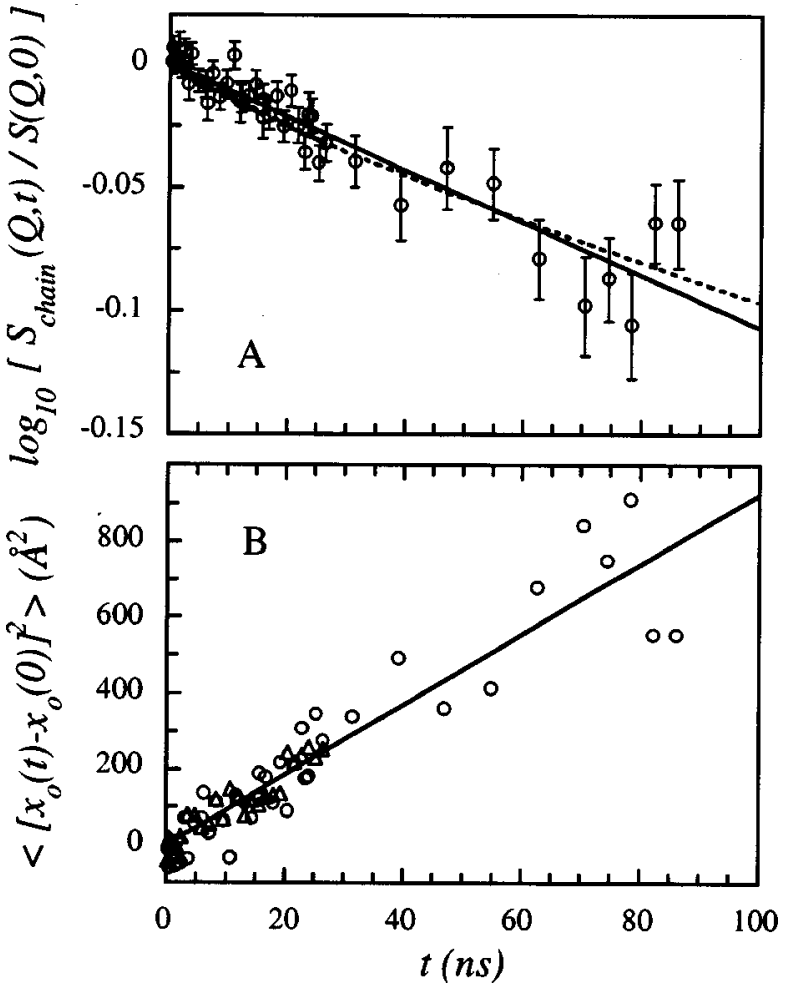

FIG. 5. (a) Logarithmic representation of the single chain structure factor at $470 \mathrm{~K}$ and $0.04 \AA^{-1}$ vs time. The solid line corresponds to a linear regression indicating single exponential decay in the diffusive regime. The dotted line shows the fit with a KWW of $\beta=0.83$. (b) Time evolution of the mean squared displacement of the center of mass of the molecule obtained from the experimental data at $0.04 \AA^{-1}(\bigcirc)$ and $0.06 \AA^{-1}(\triangle)$. The solid line shows the linear behavior corresponding to the diffusion coefficient $D_{R}$ $=1.54 \AA^{2} / \mathrm{ns}$.

nal relaxation modes are present in the dynamic structure factor. This may be deduced already from our previous phenomenological analysis of the spectra. The fit of the Rouse model $^{1}$ [Eq. (2.7) together with Eq. (2.6)] to the NSE spectra provides a good description in the $Q$ range $Q \leqslant 0.15 \AA^{-1}$ [see Fig. 6]. In this range, the elementary Rouse rate $W$ at $470 \mathrm{~K}$ is $31.6 \pm 4.4 \mathrm{~ns}^{-1}$ per main chain bond. The corresponding mode-dependent characteristic times are represented by the dashed-dotted line in Fig. 1. Taking into ac-

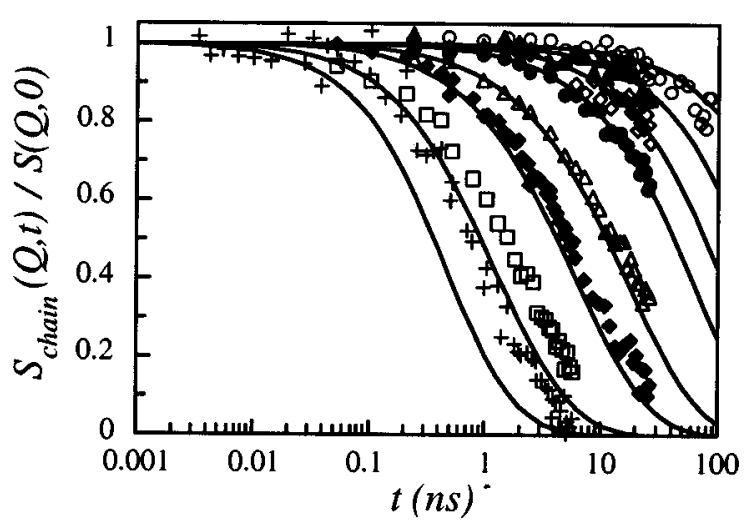

FIG. 6. Single chain dynamic structure factor at $470 \mathrm{~K}$ and $0.04 \AA^{-1}(\bigcirc)$, $0.06 \AA^{-1}(\boldsymbol{\Delta}), 0.08 \AA^{-1}(\diamond), 0.10 \AA^{-1}(\bullet), 0.15 \AA^{-1}(\triangle), 0.20 \AA^{-1}(\diamond)$, $0.30 \AA^{-1}(\square)$, and $0.40 \AA^{-1}(+)$. The solid lines show the fit with the Rouse model to the data.

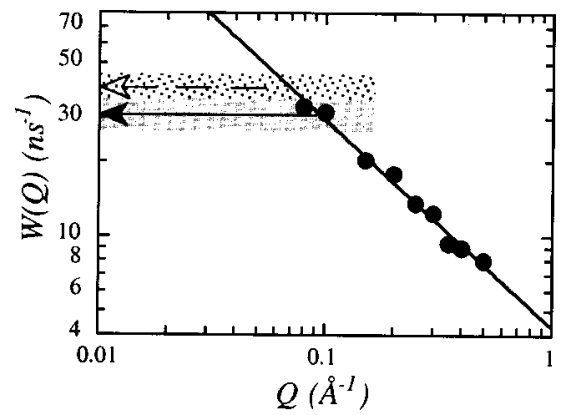

FIG. 7. Dispersion of the elementary relaxation rate per main chain bond of the chain dynamics observed in PIB. The solid arrow indicates the value obtained from the joint fit with the Rouse model to the spectra at $0.03 \AA^{-1} \leqslant Q \leqslant 0.15 \AA^{-1}$; the empty arrow, the value deduced from the diffusion coefficient obtained from the 0.03 and $0.04 \AA^{-1}$ data. The solid line represents the fit with a power law in $Q$. Shaded areas show the error bars estimated in each case.

count the relationship between $D_{R}$ and $W$, the corresponding diffusion coefficient $D_{R}$ becomes $D_{R}=1.22 \pm 0.17 \AA^{2} \mathrm{~ns}^{-1}$. Within the experimental uncertainties $D_{R}$ agrees with that deduced from the low $Q$ regime. The observation of a slightly higher value for $D_{R}$ from the analysis of the 0.03 and $0.04 \AA^{-1}$ data could also relate to some remaining contributions of the internal modes to the decay of the structure factor. Therefore, we will consider the value deduced for $D_{R}$ from the Rouse frequency as the most reliable.

Thus, the NSE results show that, within the experimental accuracy, in the $Q$ range $Q \leqslant 0.15 \AA^{-1}$ the Rouse model gives a good account for the internal modes as well as for the diffusion of the chain center of mass. However, it is also clear that for higher $Q$ values the experimental structure factors decay significantly more slowly than the Rouse model would require. In order to quantify the deviations, we fitted the Rouse model to the different spectra separately. Thereby we fixed the center of mass diffusion coefficient to $D_{R}$ $=1.22 \AA^{2} \mathrm{~ns}^{-1}$, the value obtained above, and floated the Rouse rate $W$ for the different $Q$ values. This procedure results in a strong dispersion of the elementary Rouse rate shown in Fig. 7. The values determined for $W$ at $Q$ $\geqslant 0.15 \AA^{-1}$ follow a $Q$ dependence which can be described by the power law

$$
W\left(\mathrm{~ns}^{-1}\right)=4.30 \quad Q^{-0.84} \text {, }
$$

which is depicted as a solid line in Fig. 7. Note that another origin of the slowing down of the chain relaxation compared to the Rouse prediction could be a reduction of the weights of the higher modes, which in the Rouse model are proportional to $p^{-2}$ [see Eq. (2.3)]. This analysis shows that the validity of the Rouse model in the case of PIB is restricted to the $Q$ range below $0.15 \AA^{-1}$, and some mechanism giving account for the apparent $Q$ dispersion of the elementary Rouse frequency has to be invoked in order to reproduce the experimental behavior.

\section{B. The stiffness of the chain}

As explained in Sec. II, a more realistic description than that given by the Rouse model for the dynamic structure factor in not extremely flexible polymers has to include the 


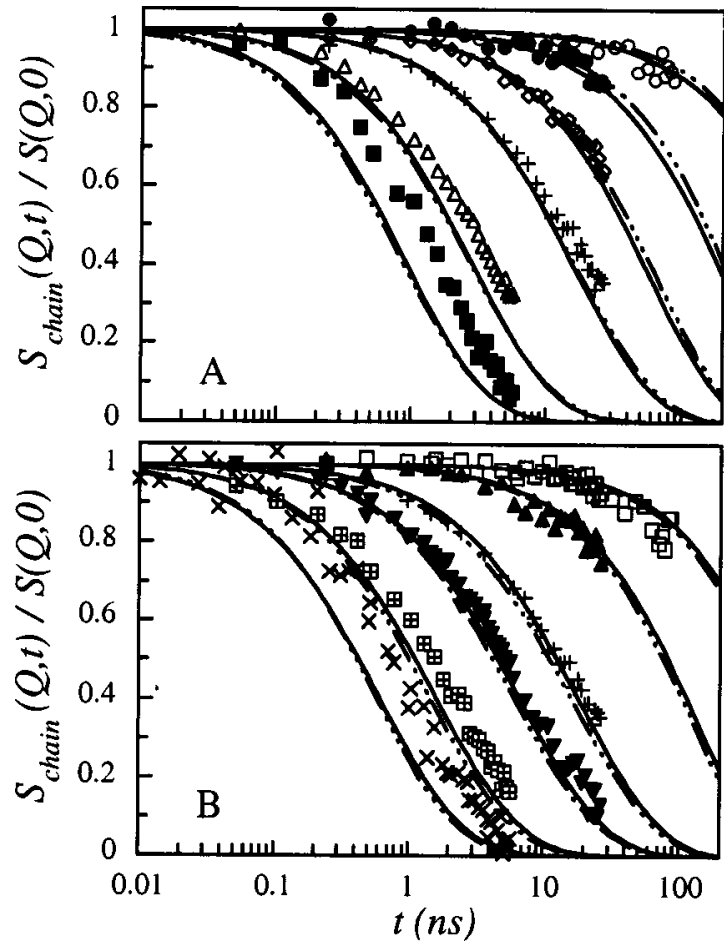

FIG. 8. (a) Comparison between the predictions of the bending forces model within the approximation of Eq. (2.6) with Eq. (2.19) (dashed-dotted lines) and the mode description (solid lines) for giving account for the stiffness of the chain, and the experimental NSE data obtained at $470 \mathrm{~K}$. (b) Comparison between the fitting curves corresponding to the bending forces model obtained through the above-mentioned approximation (dashed-dotted lines) and the full solution (solid lines) at $470 \mathrm{~K}$. The $Q$ values shown are: 0.03 $\AA^{-1}(\bigcirc), 0.04 \AA^{-1}(\square), 0.06 \AA^{-1}(\bullet), 0.08 \AA^{-1}(\mathbf{\Delta}), 0.10 \AA^{-1}(\diamond), 0.15$ $\AA^{-1}(+), 0.20 \AA^{-1}(\mathbf{\nabla}), 0.25 \AA^{-1}(\triangle), 0.30 \AA^{-1}(? ? ?), 0.35 \AA^{-1}(\mathbf{\square})$, and $0.40 \AA^{-1}(\times)$.

stiffness of the chain. The influence of the stiffness which can be expected from the characteristic ratio of PIB was calculated according to both models described in Sec. II B. For the mode description of the chain statistics we utilized $C(p)$ [Eq. (2.9)] with $C_{\infty}=6.73$ and $c=0.4$. The chain bending model was investigated both in its approximated version [Eq. (2.19)] with $\alpha_{B}=0.013$ and in terms of its full solution. Within the accuracy of the calculation both expressions are indistinguishable and lead to the same structure factors. The obtained relaxation times $\tau_{p}^{b}$ at $470 \mathrm{~K}$ show the modenumber dependence depicted in Fig. 1. They coincide for $p$ values lower than $\approx 20$ and for higher number modes the values resulting from the bending force model [Eq. (2.19)] are faster than those obtained in the other framework $\tau_{q}$ [Eq. (2.15)]. The predicted dynamic structure factors are shown in Fig. 8(a). The dashed-dotted lines correspond to the bending force approach, and the solid lines to the mode description of the chain statistics. These structure factors hardly agree better with the experimental data than that obtained from the Rouse model (Fig. 6). On the other hand, the prediction of the bending force model calculated in terms of its full solution is almost indistinguishable from that of the approximate version [see Fig. 8(b)]. It follows that the stiffness corrections to the chain motion are clearly not enough, in order to account for the experimentally observed slowing down of the relaxation.
We like to mention that a fit with the bending force model to the data allowing the variation of the parameter $\alpha_{B}$ leads to a very good agreement with the experimental spectra. However, the resulting value for $\alpha_{B}$ was 0.20 , about 15 times larger than that deduced from the SANS results, and therefore clearly unphysical.

As pointed out before, in the decay of $S(Q, t)$ each mode contributes with a weight dictated by equipartition. Since this weight is inversely proportional to $\left(p^{2}+\alpha_{B} p^{4}\right)$, a large value of $\alpha_{B}$ drastically reduces the contribution of the higher modes to the relaxation. Therefore, the good description of the experimental data by the bending force model in fitting $\alpha_{B}$ is achieved by basically suppressing the contribution of the higher modes in an artificial way. A value of 0.2 for $\alpha_{B}$ demands a characteristic ratio $C_{\infty}=26.4$ completely incompatible with the actually observed chain dimensions.

\section{Internal viscosity}

\section{Phenomenological approach to damping}

As mentioned before and shown in Fig. 7, the Rouse analysis leads to the observation of a strong $Q$ dispersion of the elemental rate $W$ which could be parametrized by the power law given by Eq. (5.2). Moreover, this result cannot be explained only by the stiffness of the polymer. A step further in modeling the chain dynamics may be performed considering in addition some kind of internal viscosity, i.e., the hindrance imposed by the intrachain potentials. Intuitively, this mechanism would cause some damping of the Rouse modes which could be the origin of the apparent $Q$ dependence of $W$. Taking Eq. (5.2) as starting point, and considering an intuitive parallelism between $Q$ vector and mode number $p$, we tentatively assume a damping mechanism which depends in form of a power law on the mode number

$$
\frac{1}{\tau_{p}^{\text {phen }}}=\frac{W \pi^{2}}{N^{2}}\left(p^{2}+\alpha_{B} p^{4}\right) p^{-\gamma},
$$

where $\gamma$ is a parameter giving account for the damping. The such defined frequencies contain the effect of both the stiffness and an internal viscosity. This would be represented by some "effective" friction coefficient $\zeta_{p} \approx p^{\gamma}$.

The chain structure factor was built with the mean squared displacements given by Eq. (2.6) substituting the relaxation times in the exponential time decay by the phenomenological $\tau_{p}^{\text {phen }}$. For the weights the dissipative $p^{-\gamma}$ factor does not play a role and the $p$ dependence remains that of the bending model. The experimental spectra were then fitted with Eq. (2.7) combined with the modified Eq. (2.6) allowing only the $\gamma$ parameter to vary - the values of $W$ and $D_{R}$ were known from the Rouse analysis of the low $Q$ regime and the value of $\alpha_{B}$ was fixed to that deduced from SANS measurements. Over the whole $Q$ range an excellent description of the experimental points was achieved for $\gamma$ $=0.67$ [Fig. 9]. This phenomenological ansatz appears to demonstrate that a mode damping mechanism is a possible answer to the so far obtained discrepancies. Thereby the retardation of the modes increases with the mode number. 


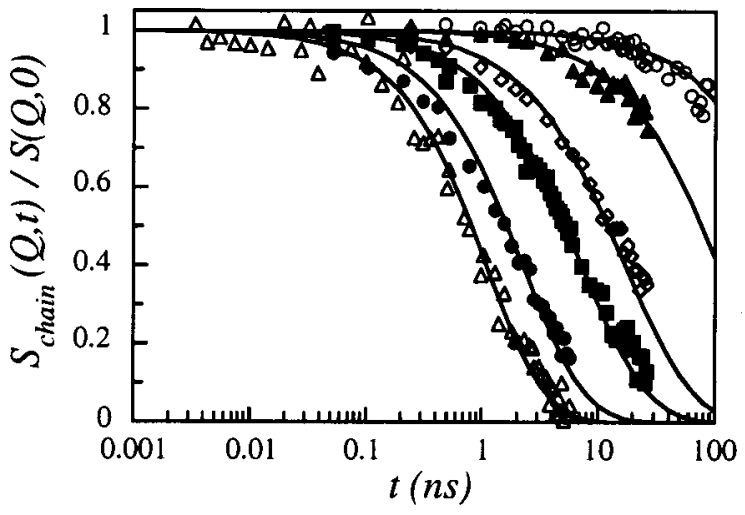

FIG. 9. Result of the description of the single chain structure factor with the phenomenological approach to the chain dynamics including a damping of the mode frequencies [Eq. (5.3)]. The data shown correspond to $470 \mathrm{~K}$ and $0.04 \AA^{-1}(\bigcirc), 0.08 \AA^{-1}(\mathbf{\Delta}), 0.15 \AA^{-1}(\diamond), 0.20 \AA^{-1}(\mathbf{\square}), 0.30 \AA^{-1}(\bullet)$, and $0.40 \AA^{-1}(\triangle)$.

\section{Allegra et al. model}

Beyond phenomenology the internal viscosity model of Allegra et al. ${ }^{5,6}$ involves a physical idea, namely the internal viscous resistance of a chain to equilibrate deviation from equilibrium. This is described in Sec. II C. Figure 10(a) presents a fit of the full solution of this model to the spectra allowing the Rouse rate $W$ and $\tau_{0}$ to float. A good agreement between theory and data was found at $470 \mathrm{~K}$ for $\tau_{0}$ $=0.28 \mathrm{~ns}$ and a slightly revised Rouse frequency of $\mathrm{W}$
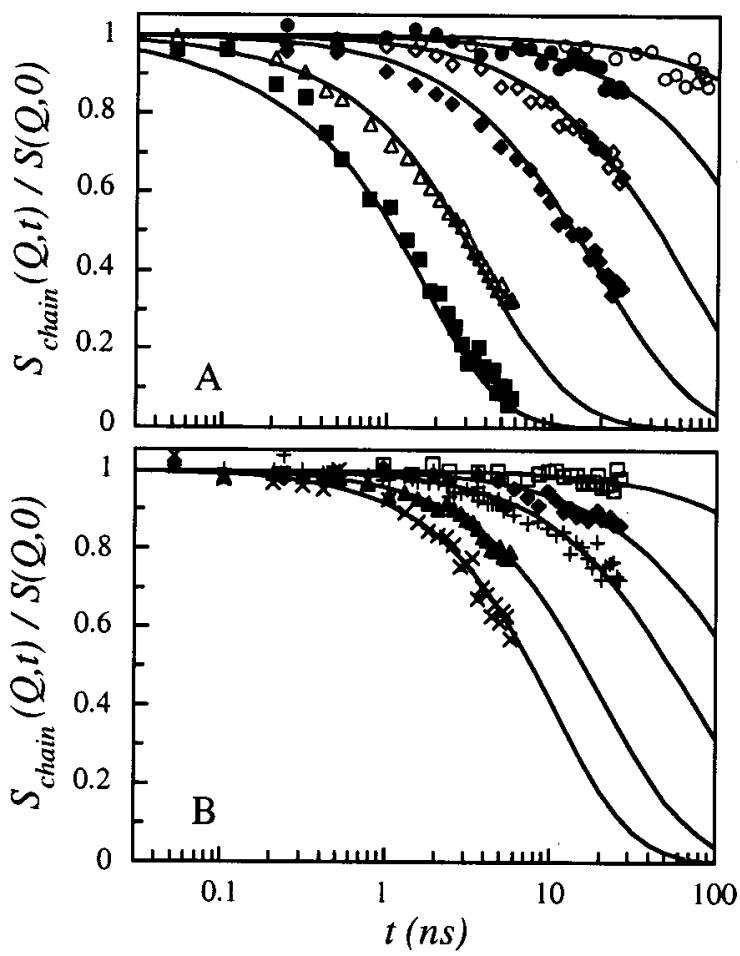

FIG. 10. (a) Fitting curves in the frame of the model proposed by Allegra et al. for different $Q$ values at $470 \mathrm{~K}$. The full solution of this model has been considered. (b) Fits to experimental data at $390 \mathrm{~K}$ with the approximation to the Allegra et al. model given in Ref. 6. The symbols correspond to: $0.03 \AA^{-1}(\bigcirc), 0.06 \AA^{-1}(\bullet), 0.08 \AA^{-1}(\square), 0.10 \AA^{-1}(\diamond), 0.15 \AA^{-1}$ $(\bullet), 0.20 \AA^{-1}(+), 0.25 \AA^{-1}(\triangle), 0.30 \AA^{-1}(\mathbf{\Delta}), 0.35 \AA^{-1}(\mathbf{\square})$, and 0.40 $\AA^{-1}(\times)$.
$=27 \mathrm{~ns}^{-1}$, although in the intermediate $Q$ range some slight discrepancies in the spectral shape are evident. Also at $390 \mathrm{~K}$ a reasonable description was possible yielding $\tau_{0}=2.5 \mathrm{~ns}$ and $W=2.54 \mathrm{~ns}^{-1}$. We note that varying the procedures to evaluate the model by, e.g., using the approximation formula mentioned in Sec. II C [as in the fitting curves shown in Fig. 10 (b) corresponding to data at $390 \mathrm{~K}$ ] or employing integral representation of the sums of the chain segments mainly changes $\tau_{0}$ while $W$ rests basically unaffected. At $470 \mathrm{~K}$ depending on the modus of evaluation $\tau_{0}$ varies between 0.19 and $0.32 \mathrm{~ns}$, while at $390 \mathrm{~K}$ the range of variation is $2.2 \leqslant \tau_{0} \leqslant 3.3 \mathrm{~ns}$. Using the above-quoted best results for $\tau_{0}$ an activation energy for the configurational change of about $0.43 \mathrm{eV}$ is found. The corresponding relaxation times $\tau_{1}$ and $\tau_{2}$ of the model at $470 \mathrm{~K}$ are displayed as solid lines in Fig. 1 . We note that for low mode numbers the phenomenological time $\tau_{p}^{\text {phen }}$ which is also presented there agrees quite well with $\tau_{1}$. Thus, the Allegra et al. model is able to underpin the phenomenological result in interpreting the "damping" in terms of the hybridization of a local and a propagating mode.

The predictions of the internal viscosity model may also be compared with the initial parametrization of the observed spectra in terms of KWW functions. For that purpose we have calculated the structure factor following from the Allegra et al. approach using the experimental parameters $W$ and $\tau_{0}$. The thus obtained theoretical spectra were fitted with KWW functions in the same way as the experimental spectra. Figure 11(a) compares the thus obtained average times $\langle\tau\rangle$ with the experimental values for 390 and $470 \mathrm{~K}$. Figure 11(b) undertakes the same test for the stretching parameter. As it was pointed out before, the general agreement is good, but differences in the shape can been found. It is noteworthy that due to the different shift factors used for the diffusion coefficient (the shift factor of 12 corresponds to an activation energy of $0.49 \mathrm{eV}$ ) and the effective relaxation time $\tau_{0}$ the model predicts a failure of the scaling. This is in particular obvious from the different $\beta$ values obtained at the same wave vector. Unfortunately, with the experimental data available at the moment it is not possible to explore this aspect in more detail.

\section{DISCUSSION AND CONCLUSION}

In this work we present the first in depth study of the changes in the dynamic structure factor of a polymer chain which occur when the Rouse regime is left toward more local length scales. The study encompasses the dynamic regimes from the translational center of mass diffusion through the Rouse regime toward local relaxation processes. It furthermore connects to recent measurements of the collective response function at higher $Q$ values. The experiments became possible due to the significant intensity gain at the spin echo spectrometer IN11 allowing for an exploration of $S(Q, t)$ at larger $Q$ where the coherent scattering intensity is low. With high quality data at hand it was possible to test different models which address the crossover phenomena to local scales mentioned above. In this section we first focus 


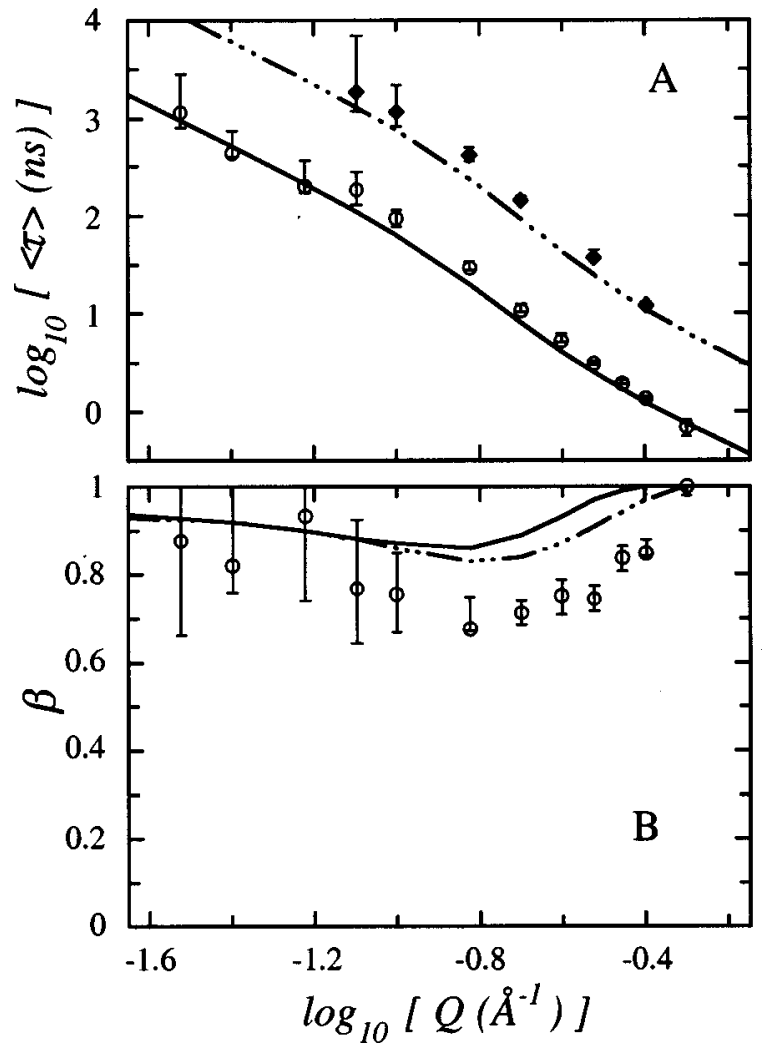

FIG. 11. Comparison between the values obtained for the average time (a) and the shape parameter (b) from the fit with a KWW to the experimental single chain dynamic structure factor (symbols) and to the theoretical fitting curves in the framework of the model proposed by Allegra et al. (lines). The temperatures investigated are $390 \mathrm{~K}(\bullet$, dashed-dotted line) and $470 \mathrm{~K}(\bigcirc$, solid line)].

on the application range of the Rouse model, then discuss the bending force model and claims related to it. Thereafter the internal viscosity approach is looked upon. Finally some recent computer simulations and the relation to the collective dynamics are addressed.

\section{A. Limit of validity of the Rouse model}

From the Rouse analysis of the spectra it turns out that this model is adequate for $Q$ values below $\approx 0.15 \AA^{-1}$. This $Q$ regime seems also to be the limit of validity of the Rouse model for describing the coherent scattering function in other investigated polymers like the very flexible polydimethylsiloxane (PDMS), and polyethylene (PE), a polymer which exhibits a similar stiffness as PIB. In the first case, it was shown ${ }^{18}$ that the internal modes of a PDMS chain were reasonably well described by the Rouse model in the regime $0.04 \leqslant Q \leqslant 0.13 \AA^{-1}$. Above this wave vector and up to 0.3 $\AA^{-1}$ the experimental results could successfully be explained incorporating the effect of the stiffness of the chain through the above-explained mode description. This indicates a much weaker influence of the internal viscosity in the case of PDMS than for PIB. In the framework of the Allegra et al. model this implies very short relaxation times for rotations around skeletal bonds. Taking into account the low value of the rotational barrier for the $\mathrm{Si}-\mathrm{O}$ bonds ${ }^{19}$ which conform the main chain in this polymer, such a behavior can be ra- tionalized. In contrast to the $\mathrm{Si}-\mathrm{O}$ bond the rotational potential around $\mathrm{C}-\mathrm{C}$ bonds which constitute most of the polymers is much higher.

Recently also the chain dynamics of PE has been investigated by NSE as well as by molecular dynamics simulations (MDS). ${ }^{2}$ The comparison between Rouse predictions and the results of both techniques lead to a reasonable agreement for $Q$ values up to $0.14 \AA^{-1}$, and for $Q \geqslant 0.18 \AA^{-1}$ the theoretical curves lie systematically below both the experimental and simulation data, and show qualitatively similar behavior to PIB.

\section{B. Bending force model}

Experimental results on the self-correlation function obtained by incoherent scattering from four different polymers including PIB [polytetrahydrofuran (PTHF), polypropylene oxide (PPO), polyethylene oxide (PEO), and PIB] were also compared with the Rouse model predictions in Ref. 20. In that work, a comparison between experiment and theory was made through the study of the $Q$ dependence of the full width at half-maximum of the incoherent scattering functions in the frequency domain. The covered $Q$ range ranged between 0.15 and $1 \AA^{-1}$. Deviations from Rouse dynamics were found in a $Q$ region which was identified with the value $\pi / 2\left(\mathbf{l}_{0} C_{\infty}\right)\left(0.52 \AA^{-1}\right.$ for PTHF, $0.39 \AA^{-1}$ for PPO and PEO, and $0.16 \AA^{-1}$ for PIB). This limit is predicted by the bending force model which was investigated in this work. However, a precise determination of this limit without the guide of any model is difficult. An unbiased inspection of the experimental results shown in Ref. 20 leads to the observation that the deviations from Rouse dynamics are stronger for PIB than for the other polymers and that in all cases (even in PIB) they occur around or below $0.5 \AA^{-1}$. Due to the limited quality of these data more precision is unfortunately not possible. The authors claimed that for all polymers in particular also for PIB an application of the bending force model could account for the experimentally determined ${ }^{21}$ characteristic $^{2}$ frequencies within the experimental uncertainties, in obvious contradiction to the findings presented here. Neither this model nor the consideration of the mode-dependent characteristic ratio without an additional damping mechanism are appropriate for describing the PIB dynamic structure factor, as has been eluded to in Sec. V.

Finally we would like to note that both the much earlier ARS approach to the stiffness problem as well as the bending force model for moderately stiff chains yield nearly indistinguishable results. This is also evident from the modenumber-dependent relaxation times of Fig. 1.

\section{Internal viscosity approach}

We have demonstrated that in the framework of the internal viscosity model of Allegra et al. a nearly quantitative description of the $Q$-dependent dynamic structure factor could be achieved. We further remark that with the phenomenological approach of a power law mode damping a very good agreement with the experiment could be achieved. This ansatz was based on the observation that the effective $Q$-dependent Rouse rate (Fig. 7) is decreasing with increas- 
ing $Q$ in form of a power law. At the end it turned out that the related relaxation times $\tau_{p}^{\text {phen }}$ for the significant lower mode numbers agree very well with the sophisticated Allegra et al. model. The phenomenological times $\tau_{p}^{\text {phen }}$ may be considered as a result of an unbiased mode analysis ${ }^{22}$ with constraints which leads to nearly identical relaxation times as the theory thereby supporting it strongly.

Now, it is interesting to compare the results obtained for the effective relaxation time for local relaxations $\tau_{0}$ with those corresponding to relaxation processes previously detected and characterized in PIB by other techniques. In this way an identification of $\tau_{0}$ with one of those processes could possibly be found. We may inspect, e.g., the temperature dependencies. For $\tau_{0}$ estimate an activation energy of about $0.43 \pm 0.1 \mathrm{eV}$. It is worthy of remark that this activation energy is considerably higher than the $\mathrm{C}-\mathrm{C}$ torsional barrier in this polymer ${ }^{23}$ (around $0.2 \mathrm{eV}$ ). The discrepancy must reflect the influence of the interactions with the environment and therefore $\tau_{0}$ appears to correspond to an effective relaxation time. Also the temperature dependence observed for the characteristic time of the Johari-Goldstein $\beta$ relaxation by dielectric spectroscopy ${ }^{13}$ (activation energy of $0.26 \mathrm{eV}$ ) is weaker than that obtained for $\tau_{0}$. On the other hand, compared to the temperature dependence of $\tau_{0}$ the activation energy of the phenomenological shift factor is higher $(0.49$ $\mathrm{eV})$. Even higher is the extrapolated rheological shift factor following Eq. (4.2), which in the temperature range of study amounts to $0.60 \mathrm{eV}$. The experimental activation energy is in the range of that for the NMR correlation time associated with correlated conformational jumps in bulk PIB by Dejean de la Batie et al. ${ }^{24}(0.46 \mathrm{eV})$ and one could tentatively relate $\tau_{0}$ to the mechanism underlying this process. However, although a similar temperature dependence is found for $\tau_{0}$ and the NMR correlation time, the obtained absolute values for $\tau_{0}$ are significantly slower. The extrapolation of the NMR time to $390 \mathrm{~K}$ amounts to $\approx 0.25 \mathrm{~ns}$, i.e., it would be around one order of magnitude faster than $\tau_{0}$ at this temperature. Apparently $\tau_{0}$ corresponds not to a single process but could be a result of a combination of local processes leading to an effective mechanism which limits the validity range of the Rouse motion in damping the relaxation modes.

\section{Computer simulation}

Recently in detailed MD simulations on the dynamics of a realistic PE chain ${ }^{2}$ it was found that in the limiting spatial range of the Rouse model the Rouse modes stretch significantly. Furthermore the simulations reveal a sublinear center of mass diffusion for $t<\tau_{R}(\beta=0.83)$. Whereas this deviation from the simple exponential behavior of the center of mass diffusion could be compatible with our experimental data, as already commented in Sec. V A and illustrated in Fig. 5(a), we do not find any hint of stretching in the internal modes. An analysis of the data with mean squared displacements evolving in a nonexponential way with time [Eq. (2.6) with a KWW function instead of the exponential] gives stretching parameters $\beta \geqslant 1$, and excludes that the stretching of the modes could serve as an explanation for the observed deviations from Rouse behavior.

\section{E. Relation to collective dynamics}

As displayed in Fig. 4(a) the average relaxation times from the collective response studied at higher $Q$ smoothly cross over to the single chain results at lower $Q$. Seemingly there exists an intimate relation between the single chain dynamic structure factor and the collective dynamics. This relation deviates from what is known from simple liquids where the single atom result is the quantity to be related with the collective response

$$
\left[S_{\text {coh }}(Q, t) \sim S(Q) S_{\text {inc }}\left(\frac{Q}{\sqrt{S(Q)}}, t\right)\right] \cdot{ }^{25}
$$

We note that according to the Rouse theory the selfcorrelation function would display considerably shorter $\langle\tau\rangle$ and thus not smoothly cross over into $\langle\tau\rangle_{\text {coll }}$. The details of these relations in polymer systems will be the subject of a future work.

In summary, observing the requirement that the conformation of the chain —as known in terms of $C_{\infty}$-must be in agreement with any bending stiffness or other related free energy contributions, it seems inevitable to assume a significant internal, i.e., intrachain friction. Whereas additional terms in the elastic free energy would reduce the high $Q$ relaxation rate by suppressing the amplitudes of fast modes (even faster than simple Rouse modes in this case), extra dissipation only affects the rates. The process causing dissipation is thought to be a delayed redistribution of the local conformations on a change of chain stretching. The activation energy connected to the rate $\tau_{0}^{-1}$ associated with the dissipation is two times the rotational energy barrier indicating some influence of the embedding melt or the necessity of correlated rotations to cause dissipation.

\section{ACKNOWLEDGMENTS}

J.C. and A.A. acknowledge support from the following projects: DGICYT, PB97-0638; GV, EX 1998-23; UPV/ EHU, 206.215-G20/98. Support from the "Donostia International Physics Center"' is also acknowledged by J.C. and A.A.

${ }^{1}$ P. E. Rouse, Jr., J. Chem. Phys. 21, 1272 (1953).

${ }^{2}$ W. Paul, G. D. Smith, D. Y. Yoon, B. Farago, S. Rathgeber, A. Zirkel, L. Willner, and D. Richter, Phys. Rev. Lett. 80, 2346 (1998).

${ }^{3}$ S. Brückner, Macromolecules 14, 449 (1981).

${ }^{4}$ P. J. Flory, Statistical Mechanics of Chain Molecules (Interscience, New York, 1969).

${ }^{5}$ G. Allegra and F. Ganazzoli, Macromolecules 14, 1110 (1981).

${ }^{6}$ G. Allegra and F. Ganazzoli, in Advances in Chemical Physics, edited by I. Prigogine and S. A. Rice (Wiley, New York, 1989), Vol. 75, p. 265.

${ }^{7}$ L. Harnau, R. G. Winkler, and P. Reineker, J. Chem. Phys. 102, 7750 (1995); 104, 6355 (1996).

${ }^{8}$ B. Ewen and D. Richter, Advances in Polymer Science (Springer, Berlin, 1967), Vol. 134.

${ }^{9}$ M. Doi and S. F. Edwards, The Theory of Polymer Dynamics (Clarendon, Oxford, 1986).

${ }^{10}$ M. Györ, Zs. Fodor, H.-C. Wang, and R. Faust, J. Macromol. Sci., Pure Appl. Chem. A31, 2053 (1994).

${ }^{11}$ J. P. Kennedy, V. S. C. Chang, R. A. Smith, and B. Ivan, Polym. Bull. (Berlin) 1, 575 (1979).

${ }^{12}$ See, e.g., Neutron Spin Echo, edited by F. Mezei, Lecture Notes in Physics, Vol. 28 (Springer, Heidelberg, 1980).

${ }^{13}$ D. Richter, A. Arbe, J. Colmenero, M. Monkenbusch, B. Farago, and R. 
Faust, Macromolecules 31, 1133 (1998).

${ }^{14}$ J. D. Ferry, Viscoelastic Properties of Polymers (Wiley, New York, 1970).

${ }^{15}$ P. G. de Gennes, Physica (Amsterdam) 25, 825 (1959).

${ }^{16}$ B. Farago et al. (unpublished).

${ }^{17}$ L. J. Fetters, D. J. Lohse, D. Richter, T. A. Witten, and A. Zirkel, Macromolecules 27, 4639 (1994).

${ }^{18}$ B. Stühn, B. Ewen, and D. Richter, Z. Phys. B 58, 305 (1985).

${ }^{19}$ I. Bahar, I. Zuniga, R. Dodge, and W. L. Mattice, Macromolecules 24, 2986 (1991).
${ }^{20}$ L. Harnau, R. G. Winkler, and P. Reineker, J. Chem. Phys. 106, 2469 (1997).

${ }^{21}$ G. Allen, J. S. Higgins, A. Maconauchie, and R. E. Ghosh, J. Chem. Soc., Faraday Trans. 2 78, 2117 (1982).

${ }^{22}$ D. Richter, L. Willner, A. Zirkel, B. Farago, L. J. Fetters, and J. S. Huang, Phys. Rev. Lett. 71, 4158 (1993).

${ }^{23}$ J. Colmenero and A. Arbe, Phys. Rev. B 57, 13508 (1998).

${ }^{24}$ R. Dejean de la Batie, F. Lauprêtre, and L. Monnerie, Macromolecules 22, 2617 (1989).

${ }^{25}$ K. Sköld, Phys. Rev. Lett. 19, 1023 (1967). 